\title{
Application of Hemoadsorption in Neonatal and Pediatric Hyperinflammatory States: A Case Series
}

\author{
Leonardo Milella ${ }^{1,}$, , Maria Teresa Ficarella ${ }^{1}$, Gerolmina Calabrese ${ }^{1}$, Michele Sisto ${ }^{1}$, \\ Rita Luana Grieco', Paola Moliterni ${ }^{1}$, Pasquale Raimondo ${ }^{1}$, Fabiana Cito ${ }^{1}$, Vito Bellino ${ }^{2}$, \\ Antonio Ranieri' ${ }^{2}$, Mario Giordano ${ }^{2}$ \\ ${ }^{1}$ Department of General Pediatric and Neonatal Anesthesia and ICU-Neonatal and Pediatric Cardiac Anesthesia and ICU, Pediatric Hospital \\ Giovanni XXIII, Bari, Italy \\ ${ }^{2}$ Department of Pediatric Nephrology and Dialysis, Pediatric Hospital Giovanni XXIII, Bari, Italy
}

Email address:

dino.milella@virgilio.it (L. Milella)

${ }^{*}$ Corresponding author

\section{To cite this article:}

Leonardo Milella, Maria Teresa Ficarella, Gerolmina Calabrese, Michele Sisto, Rita Luana Grieco, Paola Moliterni, Pasquale Raimondo, Fabiana Cito, Vito Bellino, Antonio Ranieri, Mario Giordano. Application of Hemoadsorption in Neonatal and Pediatric Hyperinflammatory States: A Case Series. American Journal of Pediatrics. Vol. 5, No. 2, 2019, pp. 34-42. doi: 10.11648/j.ajp.20190502.11

Received: February 20, 2019; Accepted: April 9, 2019; Published: May 6, 2019

\begin{abstract}
Objective: Extracorporeal blood purification therapies are increasingly applied in the field of intensive care medicine. Compared to filtration-based methods mainly used for renal replacement therapy, newest adsorptive approaches have shown to specifically target the inflammatory cascade by the effective removal of relevant mediators. In the neonatal and pediatric setting however, the application of these methods brings with it various challenges but also profound technical difficulties. Recently, a promising extracorporeal device for cytokine adsorption (CytoSorb) was introduced. However, data for its application in critically ill pediatric patients remains sparse. Design: Single center retrospective case study; Setting: Tertiary neonatal and pediatric general intensive care unit; Patients: The study comprised 10 critically ill pediatric patients; Interventions: We describe the use of CytoSorb in combination with standard therapy, continuous renal replacement therapy (CRRT) and plasmapheresis in ten severely ill pediatric patients with multiple organ failures of various etiologies; Measurements: The aim was to assess the effects on the inflammatory status, hemodynamics, and clinically relevant outcome parameters as well as the feasibility and safety of CytoSorb application in pediatrics; Main Results: We observed a marked decrease in inflammatory mediators, a reduction in catecholamine dosages and an improvement in organ functions, which was particularly pronounced in patients who survived. An early onset of treatment (at best within 24-48 hours after diagnosis of sepsis) seemed to be beneficial for eventual survival. Conclusions: The present case series is the first documentation of a set of pediatric/neonatal patients in which a combined therapeutic approach of hemoadsorption and renal replacement therapy showed promising results with regard to hemodynamic stabilization, control of the inflammatory response, improvement in organ functions as well as safety and feasibility. Further prospective randomized controlled studies in the pediatric field are necessary to elucidate the full potential of hemoadsorption in this set of patients.
\end{abstract}

Keywords: Multiple Organ Failure, Pediatrics, Hyperinflammation, Plasmapheresis, Hemoadsorption, Cytosorb

\section{Introduction}

The application of extracorporeal blood purification techniques is gaining importance in the intensive care field for the adjunctive treatment of sepsis and septic shock with multiple organ failure (MOF) of various origins [1]. These techniques are, increasingly used for the treatment of clinical pathological states such as acute respiratory distress syndrome (ARDS), endocarditis, hematological diseases, and neurological inflammatory pathologies of both, viral or bacterial etiology. Moreover, clinical procedures i.e. prolonged cardiopulmonary bypass (CPB), extracorporeal membrane oxygenation (ECMO) and ventricular assist devices (VAD), whose application is per se associated with stimulation of the inflammatory cascade 
which significantly affects morbidity, represent valid and increasingly entered fields of application [2,3].

High volume hemofiltration (HVHF) and hemodialysis (continuous veno-venous hemodialysis - CVVHD), both intended as renal replacement therapies, have been shown to improve various physiological outcomes (i.e. hemodynamics) including a decrease in vasopressor demand, improvement in sepsis-related metabolic acidosis and coagulopathies in septic shock with MOF [4].

Additionally, therapeutic plasmapheresis also represents a valid approach to reduce potentially toxic levels of specific compounds. However based on experience to date, its use is limited to rather specific fields of application such as Goodpasture syndrome, multiple sclerosis, Guillain-Barré syndrome, thrombotic thrombocytopenic purpura, and in hemolytic uremic syndrome [5].

In the neonatal and pediatric settings however, the application of these methods brings with it various challenges with regards to profound technical difficulties, the response variability of the cardiovascular system, substantial differences in the dynamics of production and maintenance of circulating inflammatory mediator levels, the limited ability to remove cytokines, as well as the need for high volumes of replacement fluids triggering hematologic rheology alterations [6]. The latter is associated with problems in patients with a small circulating volume (such as pediatrics) and in conditions of low cardio-circulatory reserve to maintain adequate flow.

The recent introduction of an extracorporeal device for cytokine adsorption, namely CytoSorb (Cytosorbents Europe, $\mathrm{GmbH}$, Berlin, Germany), which was approved in Europe for the treatment of hyperinflammation in patients weighing not less than $40 \mathrm{~kg}$, has gained interest in the fields of general and cardiothoracic intensive care medicine as well as in cardiac surgery and more generally in all conditions where inflammatory mediators are elevated and therefore an uncontrolled systemic inflammatory response with the risk of development of multiple organ failure prevails [7].

From our limited experience in neonatal and pediatric patients, CytoSorb has also proved useful in modulating the inflammatory response as a consequence of using extracorporeal assist devices, allowing for a better control not only of inflammation, but of the vast majority of pathological and severely compromised metabolic states related to multiple organ dysfunction syndrome [8].

Herein we describe 10 critically ill pediatric patients with multiple organ failure from various etiologies including septic shock and cardiac failure, who were treated with hemoadsorption using CytoSorb. The aim of this work was to assess the effects on inflammatory status, hemodynamics, and clinically relevant outcome parameters as well as the feasibility of CytoSorb application in pediatrics and to report on potential safety issues in this group of patients.

\section{Material and Methods}

This case series was conducted in the Neonatal and Pediatric General Intensive Care and Intensive Cardiac and Neonatal
Cardiac Surgery at the Pediatric Hospital Giovanni XXIII, Bari, Italy. Informed consent for retrospective data evaluation was obtained from all patients' parents, including an accurate description of the severity of the clinical picture, the hitherto ineffectiveness of standard therapies justifying the need for the use of CytoSorb, and the operating principles of the adsorber. CytoSorb was applied without the approval of an ethics committee. For all other procedures a prior authorization remains valid (ETHICAL COMMITTEES 53891- 2017).

From May 2016 to April 2018, a total of 10 pediatric patients inhomogeneous for age, weight, pathology and therapeutic treatment were treated and monitored at our institution.

In brief, all patients had multiple organ failure of various etiologies including septic shock and cardiac failure (Table 1) and were refractory to conventional therapeutic treatment. Hemodynamic management with catecholamines (i.e. 0.01-0.2 $\mu \mathrm{g} / \mathrm{kg} / \mathrm{min}$ epinephrine, $0.03-0.4 \mu \mathrm{g} / \mathrm{kg} / \mathrm{min}$ norepinephrine, milrinone) and volume therapy (20 to $60 \mathrm{ml} / \mathrm{kg}$ with crystalloids and fresh frozen plasma, as well as $20 \%$ sodium albumin at $2 \mathrm{ml} / \mathrm{h}$ in continuous infusion) was performed according to the standard of care protocol. All patients were intubated and mechanically ventilated. In the presence of refractory heart failure, immediate extracorporeal life support (ECLS) was initiated (in $\mathrm{n}=5$ patients, see Table 1). All patients received broad-spectrum antimicrobial therapy with III and IV generation cephalosporins, carbapenems, vancomycin and aminoglycosides. In the presence of concomitant mycotic or viral infection, antifungal (caspofungin) or antiviral (acyclovir) therapy was established, respectively. If the patient had minimum acute kidney injury (AKI) stage II criteria (serum creatinine $>2$-fold the basic value, urine output $<0.5 \mathrm{ml} / \mathrm{kg} / \mathrm{h}$ for $\geq 12$ hours, glomerular filtration rate (GFR) $<90 \mathrm{ml} / \mathrm{h}$ ), CRRT was commenced.

CRRT was performed using a Prismaflex device (Gambro, Germany) together with an ST 60 filter run in continuous veno-venous hemodiafiltration (CVVHDF) mode with a blood priming and dialysis flow ranging from $20-30 \mathrm{ml} / \mathrm{kg} / \mathrm{h}$ and an ultrafiltration rate of 10 to $100 \mathrm{ml}$, heparin anticoagulation $(5-30 \mathrm{IU} / \mathrm{Kg} / \mathrm{h})$ targeting an activated clotting time (ACT) between 150-200 sec, and an APTT-INR between 1.5-1.8. After priming of the CVVHDF circuit with normal saline, blood was used to prefill the extracorporeal CVVHDF circuit (in average $100-200 \mathrm{ml}$ ) plus additional $90 \mathrm{ml}$ to fill the CytoSorb cartridge and adapters. In case of a high hematocrit, plasma ( $10 \mathrm{ml} / \mathrm{kg}$ body weight) was infused throughout the procedure instead of blood.

Additionally, as standard dosage a continuous infusion of furosemide at $10 \mathrm{mg} / \mathrm{h}$ was started in all patients and increased to $25 \mathrm{mg} / \mathrm{h}$ if required. All variables (priming volume, flow and ultrafiltration rate) were corrected for and guided by weight, the patient's body surface, creatinine levels, glomerular filtrate rate (GFR) and evidence of cell membrane leakage syndrome (i.e. presence of peripheral edema and ascites, increase in abdominal girth or the head circumference especially in newborns and toddlers).

Therapeutic albumin exchange and plasmapheresis (to reduce the need and volume of transfusion) was initiated in 
six patients (pts no $1,2,3,5,6,7$ ) and performed every 24 hours, each for 4 hours, with temporary suspension of CRRT, using a filter set (Prismaflex TPE 1000) with blood priming and exchange of $8-100 \mathrm{ml} / \mathrm{h}$ of albumin and plasma.

If there was no improvement in the clinical condition after 24 $-48 \mathrm{~h}$ maximum a CytoSorb adsorber was used as an adjuvant treatment option in addition to the use of therapeutic albumin exchange and plasmapheresis connected as explained above [8]. The CytoSorb adsorber was integrated in a post-hemofilter position within the standard CVVHDF circuit (Prismaflex System, Germany) run at blood flows of $200 \mathrm{ml} / \mathrm{min}$; however in one case it's was placed into the blood autotransfusion circuit of the operative field (autotrans) during a Fontan's intervention. Therapeutic albumin exchange and plasmapheresis were not performed in this patient before initiation of CytoSorb due to prolonged cardiopulmonary bypass duration associated with the onset of hyperinflammation and MOF. Treatments with CytoSorb were planned to have a minimum duration of 17 hours and a maximum duration of 24 hours (recommended application duration by the company is 24 hours), separated from each other by a 24 hour pause interval to verify potential improvements in Pediatric Risk of Mortality score (PRISM III) and to analyze cytokine levels in an external laboratory, while CVVHDF was continued. Patients underwent multiple treatments (planned between 1-3) based on their clinical response, as evidenced by cardiovascular stabilization ( $>50 \%$ reduction in catecholamines) compared to start of treatment. Further target parameters
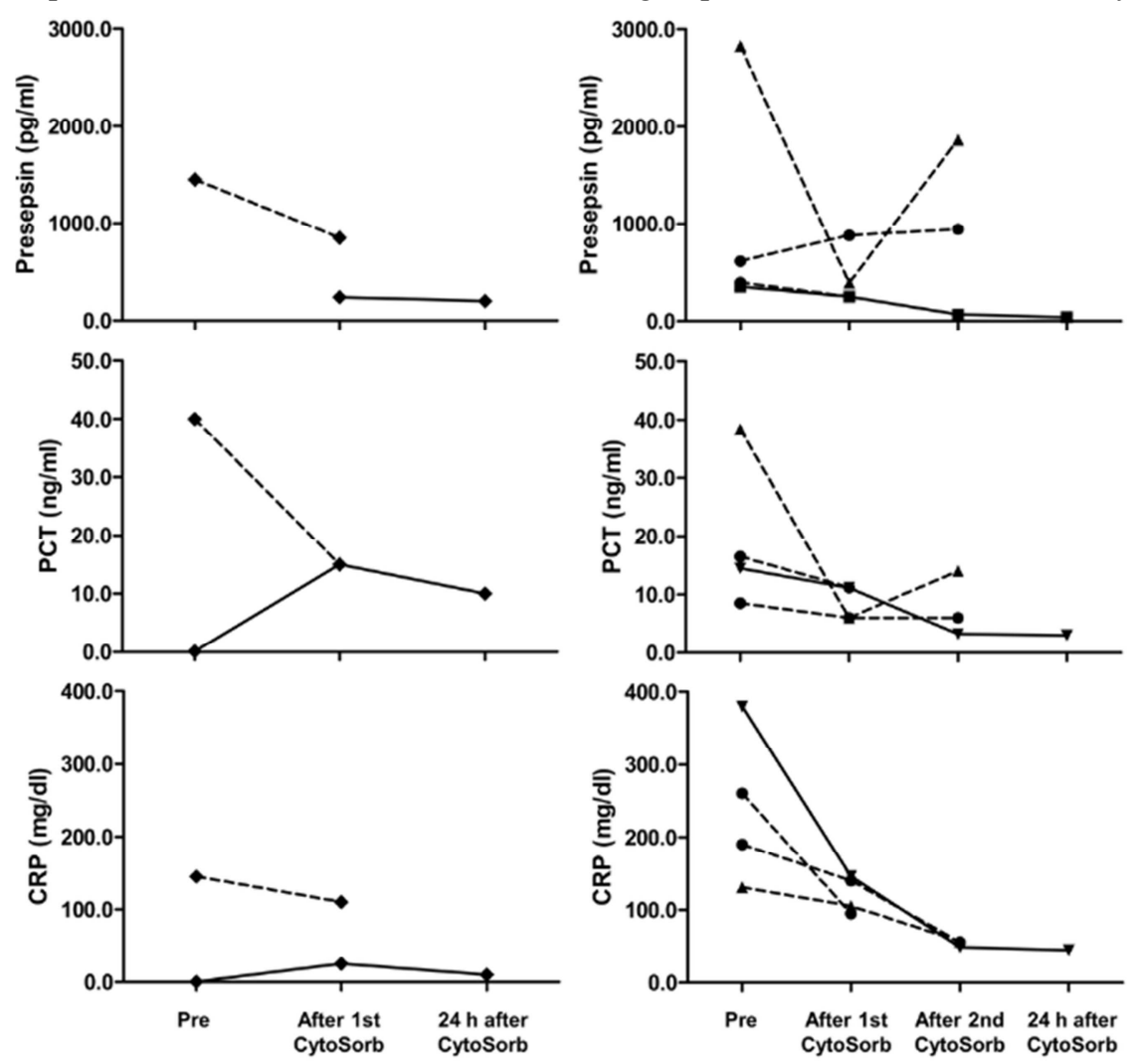

included adequate blood pressure with a mean arterial pressure $\geq 70 \mathrm{mmHg}$, central venous pressure of $7-8 \mathrm{mmHg}$ and resolution of lactic acidosis and of bicarbonate deficit.

To assess the therapeutic impact of the hemoadsorption treatment, we measured laboratory parameters of inflammation (i.e. CRP, PCT, presepsin, TNF $\alpha$, IL-6, IL-10), free hemoglobin, myoglobin and PRISM III scores throughout the treatment period (PRISM III score until recovery). Furthermore, we assessed the extent of catecholamine therapy and organ support (days on mechanical ventilation, ECMO, CRRT). ICU length of stay and ICU survival were obtained for determination of outcome.

\section{Results}

Patient characteristics and diagnoses were rather heterogeneous (Table 1). Patient age ranged from 1-312 months (median 33) and patient weight in the study group was between 3.5 - $52 \mathrm{~kg}$ (median weight $19 \mathrm{~kg}$ ), 7 patients were female, 3 were male. All pediatric patients showed a clinical condition characterized by the presence of a dysregulated systemic inflammatory response and uncontrolled release of inflammatory mediators (Figure 1 and 2) and all were in an advanced state of refractory septic shock or cardiac failure with a dysfunction of two or more organs (including CRRTdependent renal failure), DIC and severe cardio-circulatory and metabolic dysfunctions (Table 1).

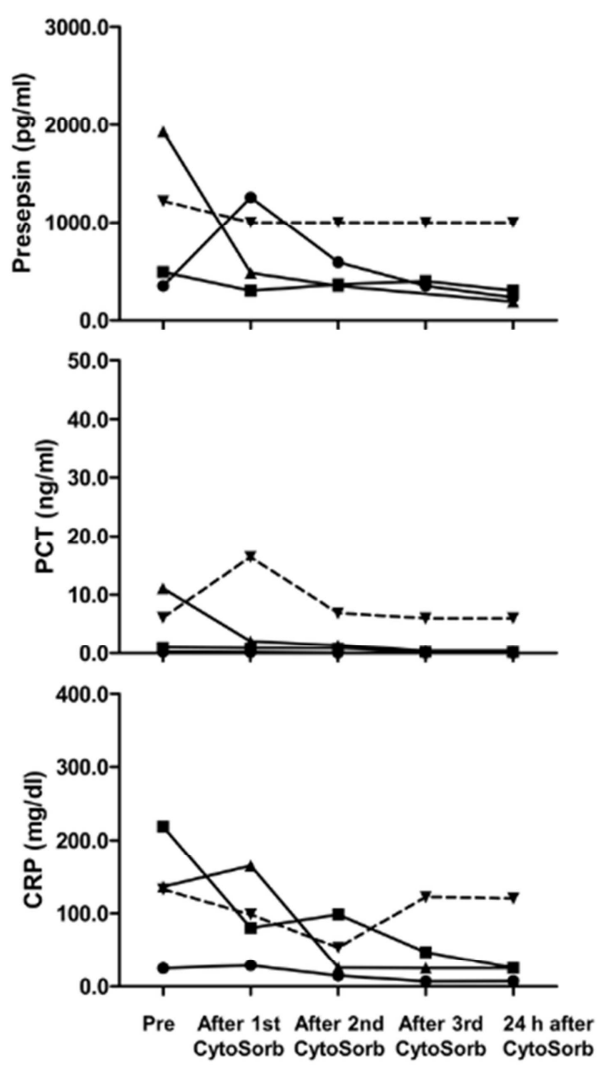

Figure 1. Levels of presepsin, PCT and CRP during the course of treatment. From left to right images for each parameter display patients receiving 1 (left), 2 (middle) or 3 (right) CytoSorb treatments, respectively. Values were assessed directly prior to treatment (pre), immediately after termination of each treatment (after CytoSorb) and 24 hours after termination of the last treatment (24 hours after CytoSorb). Solid lines indicate ICU survivors, dashed lines represent ICU non-survivors. Please note that some data were not available from all patients. 

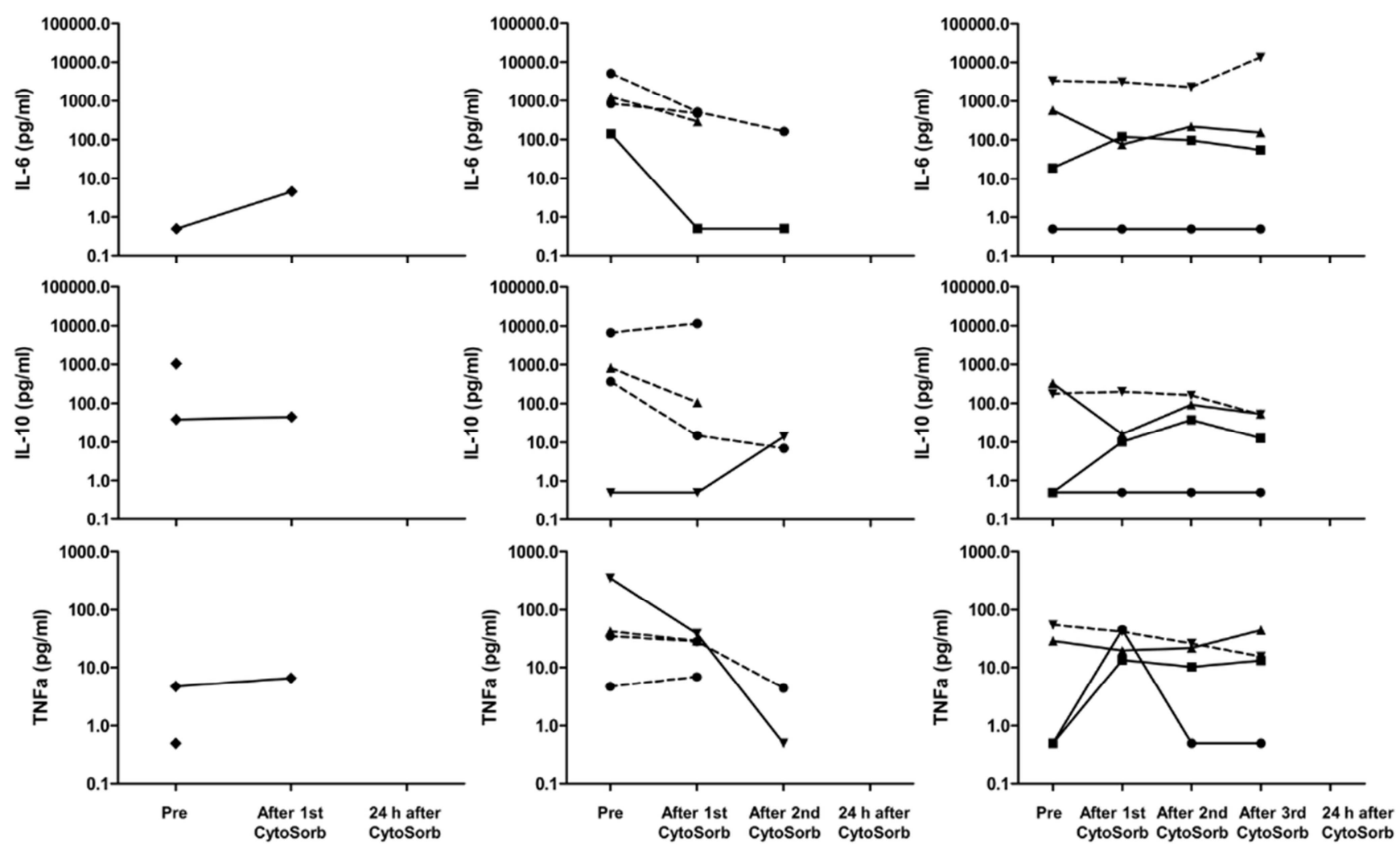

Figure 2. Levels of IL-6, IL-10, and TNF $\alpha$ during the course of treatment. From left to right images for each parameter display patients receiving 1 (left), 2 (middle) or 3 (right) CytoSorb treatments, respectively. Values were assessed directly prior to treatment (pre), immediately after termination of each treatment (after CytoSorb) and 24 hours after termination of the last treatment (24 hours after CytoSorb). Solid lines indicate ICU survivors, dashed lines represent ICU non-survivors. Please note that some data were not available from all patients.

Table 1. Patient characteristics, treatment modalities and patient outcome.

\begin{tabular}{|c|c|c|c|c|c|c|}
\hline Case & Age (months) & Gender & Weight (kg) & BMI & Diagnosis & Microbiological findings \\
\hline 1 & 1 & M & 3.5 & 14 & RVAPT & Candida parapsilosis \\
\hline 2 & 82 & $\mathrm{~F}$ & 16 & 13.2 & ARDS, Sepsis & $\begin{array}{l}\text { Pseudomonas aeruginosa, Candidas albicans, Klebsiella } \\
\text { pneumoniae, Torulopsis glabrata }\end{array}$ \\
\hline 3 & 48 & $\mathrm{~F}$ & 19 & 19 & HLH, Sepsis & Clostridium difficilis, Streptococcus pneumoniae \\
\hline 4 & 126 & $\mathrm{~F}$ & 35 & 17.8 & Acute myocarditis & Streptoccoccus pneumoniae \\
\hline 5 & 17 & $\mathrm{~F}$ & 10 & 13.8 & HUS, septic shock & Escherichia coli $\mathrm{O} 157$ \\
\hline 6 & 312 & $\mathrm{~F}$ & 52 & 21.6 & Acute myeloid leukemia, sepsis & Clostridium difficile, Candida krusei, HHV6 \\
\hline 7 & 60 & M & 25 & 21 & ADEM & HHV7, Acinetobacter baumannii, Haemoliticus spec. \\
\hline 8 & 11 & M & 10.6 & 21.6 & Septic arthritis, sepsis & $\beta$-hemolytic streptococcus, Staph aureus \\
\hline 9 & 6 & $\mathrm{~F}$ & 8 & 22 & $\begin{array}{l}\text { Fontan procedure complicated by } \\
\text { severe bleeding and prolonged } \\
\text { cardiopulmonary bypass }\end{array}$ & $\begin{array}{l}\text { none (Cytosorb used during prolonged extracorporeal } \\
\text { circulation) }\end{array}$ \\
\hline 10 & 9 & $\mathrm{~F}$ & 4.8 & 17.5 & CAVC, sepsis & Proteus mirabilis, Candida parapsilosis, Metapneumovirus \\
\hline
\end{tabular}

\begin{tabular}{|c|c|c|c|c|c|c|}
\hline Case & No of organs failures & $\begin{array}{l}\text { No of CytoSorb } \\
\text { treatments }\end{array}$ & $\begin{array}{l}\text { CytoSorb treatment } \\
\text { durations }\end{array}$ & $\begin{array}{l}\text { Delay CytoSorb therapy } \\
\text { start (hours after } \\
\text { diagnosis of sepsis) }\end{array}$ & $\begin{array}{l}\text { Specific CytoSorb } \\
\text { priming procedure } \\
\text { Y/N }\end{array}$ & $\begin{array}{l}\text { Mechanical } \\
\text { ventilation } \\
\text { (days) } \\
\end{array}$ \\
\hline 1 & $\begin{array}{l}\text { Hem, Ren, Res, Hep, Coa, } \\
\text { Neu }\end{array}$ & 1 & 19 hours & $>48$ hours & $\mathrm{Y}$ & 38 \\
\hline 2 & Hem, Ren, Res & 3 & 54 hours (19 h per treatment) & 24-48 hours & $\mathrm{Y}$ & 22 \\
\hline 3 & Hem, Ren, Res, Coa & 3 & 60 hours $(20 \mathrm{~h}$ per treatment $)$ & $<24$ hours & Y & 4 \\
\hline 4 & Hem, Ren, Res, Neu & 2 & $48 \mathrm{~h}(24 \mathrm{~h}$ per treatment $)$ & $>48$ hours & $\mathrm{Y}$ & 6 \\
\hline 5 & Hem, Ren, Res, Coa, Neu & 2 & $36 \mathrm{~h}$ (18 h per treatment) & $>48$ hours & $\mathrm{Y}$ & 4 \\
\hline 6 & Hem, Ren, Res, Hep, Neu & 2 & 38 hours ( 19 h per treatment) & $>48$ hours & $\mathrm{Y}$ & 8 \\
\hline 7 & Hem, Ren, Res, Neu & 3 & 57 hours (19 h per treatment) & 24-48 hours & $\mathrm{Y}$ & 29 \\
\hline 8 & Hem, Ren, Res, Coa & 2 & 48 hours ( $24 \mathrm{~h}$ per treatment) & $<24$ hours & $\mathrm{Y}$ & 5 \\
\hline 9 & Hem, Ren, Res, Coa & 1 & 6 hours & $<24$ hours & $\mathrm{Y}$ & 4 \\
\hline 10 & Hem, Ren, Res, Coa, Hep & 3 & 52 hours ( $17 \mathrm{~h}$ per treatment) & 24-48 hours & $\mathrm{Y}$ & 21 \\
\hline
\end{tabular}




\begin{tabular}{|c|c|c|c|c|c|c|}
\hline Case & $\begin{array}{l}\text { Catecholamines } \\
\text { (days) }\end{array}$ & $\begin{array}{l}\text { ECMO } \\
\text { (days) }\end{array}$ & $\begin{array}{l}\text { CRRT } \\
\text { (days) }\end{array}$ & $\begin{array}{l}\text { ICU LOS } \\
\text { (d) }\end{array}$ & $\begin{array}{l}\text { ICU survival } \\
(\mathbf{y} / \mathbf{n})\end{array}$ & Comment \\
\hline 1 & 38 & 20 & 20 & 38 & NO & The patient died after 38 days on ICU from cardiac failure \\
\hline 2 & 5 & 0 & 2 & 17 & YES & Survival and discharged from hospital \\
\hline 3 & 8 & 0 & 5 & 14 & YES & Survival and discharged from hospital \\
\hline 4 & 6 & 6 & 6 & 6 & NO & The patient died after 6 days on ICU from encephalitis and cerebral death \\
\hline 5 & 4 & 2 & 8 & 4 & NO & The patient died after 4 days on ICU from irreversible cardiac failure \\
\hline 6 & 7 & 0 & 2 & 7 & NO & $\begin{array}{l}\text { The patient died after } 7 \text { days on ICU from septic shock (Cytosorb applied } \\
\text { too late }>48 \mathrm{~h} \text { ) }\end{array}$ \\
\hline 7 & 12 & 0 & 3 & 23 & YES & Survival and discharged from hospital \\
\hline 8 & 2 & 0 & 3 & 6 & YES & Survival and discharged from hospital \\
\hline 9 & 4 & 1 & 0 & 7 & YES & Survival and discharged from hospital \\
\hline 10 & 10 & 16 & 7 & 30 & NO & $\begin{array}{l}\text { The patient died after } 30 \text { days on ICU (due to non septic reasons, i.e. } \\
\text { massive aortic valve insufficiency) }\end{array}$ \\
\hline
\end{tabular}

Res: respiratory

Hem: Hemodynamic

Ren: renal

Hep: hepatic

Coa: Coagulation

Neu: Neurologic

RVAPT: Total anomalous venous pulmonary return

HLH: Hemophagocytic lymphohistiocytosis

HUS: Hemolytic uremic syndrome

ADEM: Acute disseminated encephalomyelitis

CAVC: Complete atrioventricular canal defect
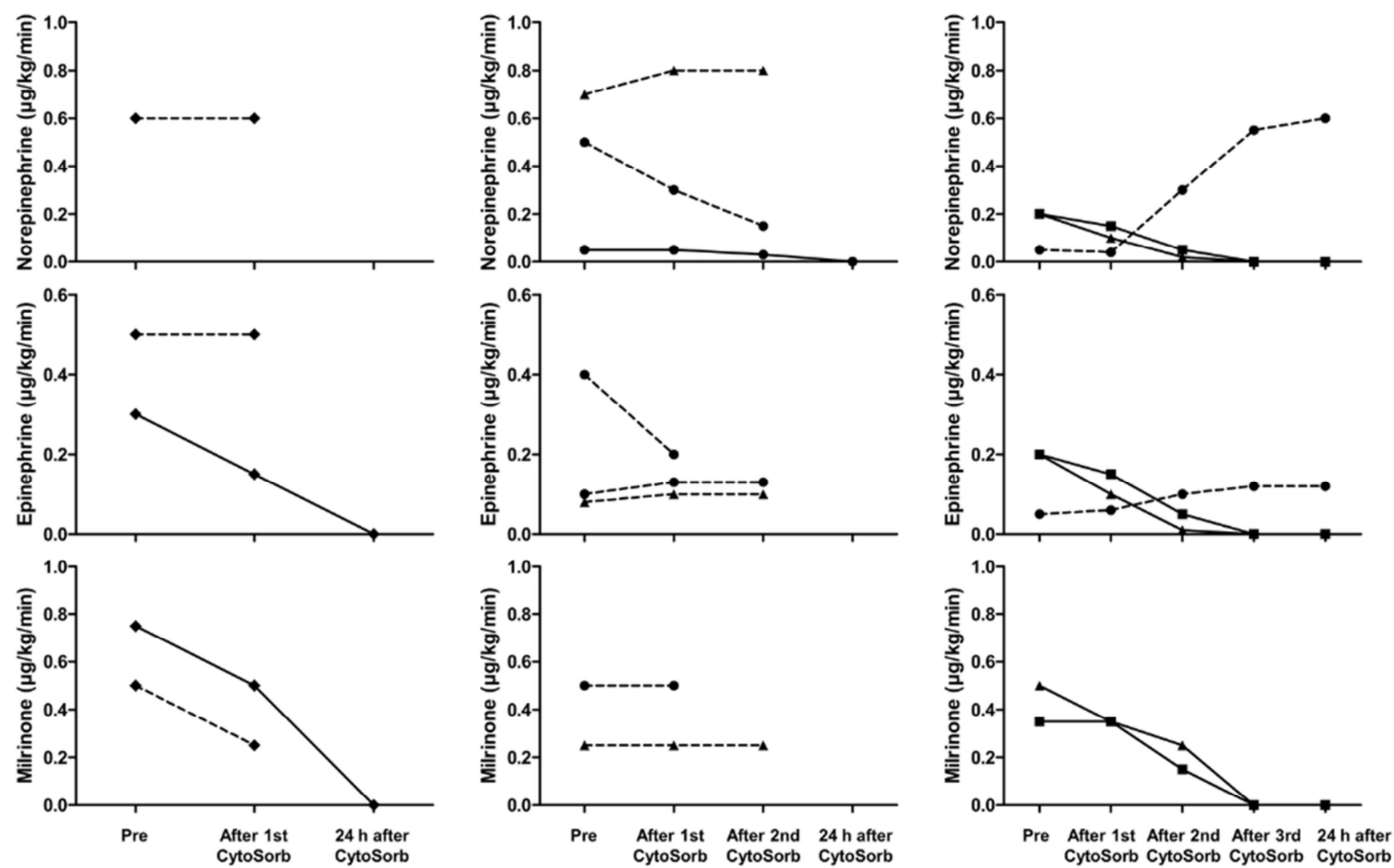

Figure 3. Development of catecholamine need during the course of treatment. From left to right images for each parameter display patients receiving 1 (left), 2 (middle) or 3 (right) CytoSorb treatments, respectively. Values were assessed directly prior to treatment (pre), immediately after termination of each treatment (after CytoSorb) and 24 hours after termination of the last treatment (24 hours after CytoSorb). Solid lines indicate ICU survivors, dashed lines represent ICU non-survivors. Please note that some data were not available from all patients. 

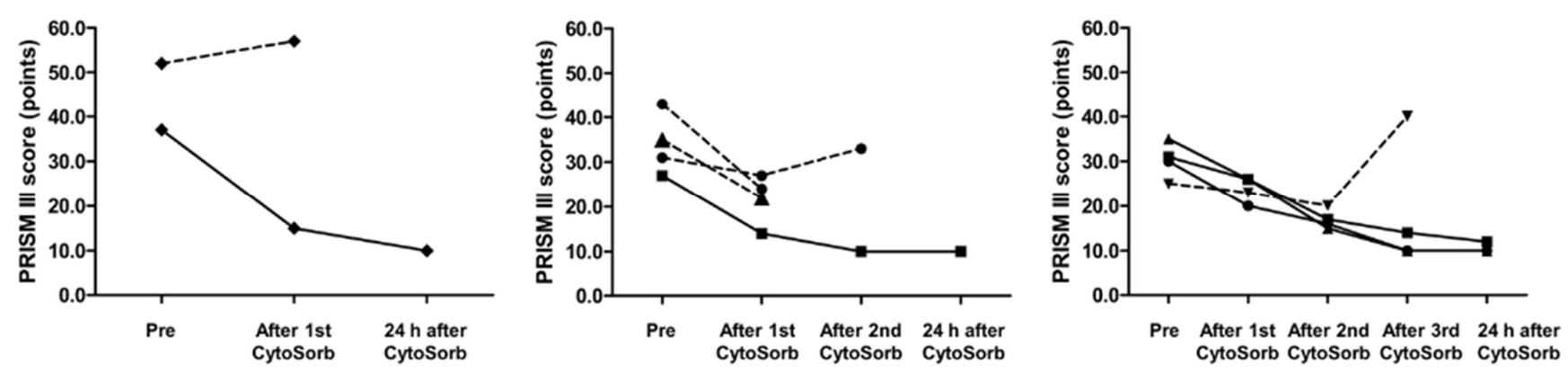

Figure 4. PRISM III scores during the course of treatment. From left to right images for each parameter display patients receiving 1 (left), 2 (middle) or 3 (right) CytoSorb treatments, respectively. Values were assessed directly prior to treatment (pre), immediately after termination of each treatment (after CytoSorb) and 24 hours after termination of the last treatment (24 hours after CytoSorb). Solid lines indicate ICU survivors, dashed lines represent ICU nonsurvivors. Please note that some data were not available from all patients.

All patients had CytoSorb treatments that ranged from 1 up to 3 (median 2) sessions. Three of the 5 survivors required 3 CytoSorb treatments to obtain clinical stabilization while a fourth patient who received 3 treatments and survived the acute phase eventually died after 30 days in ICU (due to nonseptic reasons, i.e. massive aortic valve insufficiency) (Table 1). In all patients except for four cases (1. a patient in which CytoSorb was used at an early stage, 2. a case of ECMO for cardiogenic shock and 3. a case of prolonged intraoperative cardiac bypass, 4.a case of RVPT and septic shock), the use of CytoSorb was preceded by three sessions of therapeutic albumin exchange and plasmapheresis and CytoSorb was used as last resort therapy.

Figure 1 and 2 show presepsin, PCT and CRP, TNF $\alpha$, IL-6 and IL-10 levels prior to treatment (pre), after each CytoSorb treatment and 24 hours after the last CytoSorb treatment depending on the number of therapy sessions. The combined treatment with CVVHDF, therapeutic plasmapheresis and CytoSorb was associated with a marked decrease in all inflammatory mediators (Figure 1,2). After cessation of treatment, levels persisted at the lower level for more than 24 hours (Figure 1, 2). Eventual non-survivors showed generally higher pre-treatment levels compared to survivors. While plasma concentrations of survivors were gradually reduced during the course of the combined treatments, levels of nonsurvivors tended to remain constant or even increased over time (Figure 1, 2).

We further observed a reduction in catecholamine (norepinephrine, epinephrine) and milrinone dosages at the same time (Figure 3), which was particularly evident in the group that survived. Non-survivors required higher dosages and a broader spectrum of combined catecholamine and milrinone support.

Again, non-survivors started at a higher level of catecholamine support compared to survivors and levels could not be reduced sufficiently to achieve hemodynamic stabilization or to avoid premature death. We did not observe a recurrence for catecholamine (norepinephrine, epinephrine) nor for milrinone requirement 24 hours after termination of CytoSorb treatment in the group that survived.

No patient treated with hemoadsorption with a delay of more than 48 hours from diagnosis $(n=4)$ survived, one patient with a therapy delay between $24-48$ hours died (as outlined above). All patients who started their hemoadsorption treatment within 24 hours after diagnosis $(n=3)$ survived as did two patients treated between 24-48 hours after diagnosis of sepsis (Table 1).

PRISM III score appeared lower in patients treated early (delay from diagnosis between 24-48h, median 20h) compared to patients treated $>48$ hours (median 22). Moreover, PRISM III scores progressively decreased during the course of treatments, predominantly in the group that survived (Figure 4).

Five of the 10 patients reported in our case series eventually died during their intensive care stay (Table 1). Two of the nonsurviving patients died after more than 30 days on ICU. One patient died from cardiac failure, one from encephalitis and cerebral death, and one due to massive aortic valve insufficiency. Another patient died after 7 days in ICU from septic shock, with a delay of more than 48 hours. The $5^{\text {th }}$ patient died after 4 days in ICU from irreversible cardiac failure.

CytoSorb was safely used in the 10 pediatric patients described herein and was well tolerated, with no specific device-related adverse events occurring during or after treatment. In all treated patients, a specific CytoSorb priming procedure was applied. i.e. blood or plasma was used as priming fluid (instead of normal saline) due to the restricted blood volume of pediatric patients (Table 1). Hemoadsorption treatment using CytoSorb was easy to implement and worked well in combination with simultaneous extracorporeal CVVHDF/plasmapheresis therapy.

\section{Discussion}

In the presented case series we describe the combined use of standard of care management, renal replacement therapy, therapeutic plasmapheresis and hemoadsorption therapy using CytoSorb, to treat 10 critically ill pediatric patients presenting multiple organ failure of various etiologies including septic shock and cardiac failure, who were refractory to conventional therapeutic treatment. The effects of treatment on the inflammatory status, hemodynamics, and on clinically relevant outcome parameters were assessed. In survivors we observed a marked decrease in all inflammatory mediators measured and a reduction in catecholamine dosages, while eventual non-survivors showed higher initial 
levels of inflammatory mediators and generally required higher dosages and a broader spectrum of combined catecholamine support compared to survivors. The early onset of treatment (at best within 24-48 hours after diagnosis of sepsis) seemed to be beneficial for eventual survival.

There are many pathologies that can lead to an abnormal response of the immune system including congenital disorders, infections, trauma and pathologies induced by operative procedures. In the pediatric patient, these issues seem even more difficult to handle considering peculiarities such as underlying pathologies, organ maturation and their functionality, congenital and/or acquired alterations in immunity and the inflammatory response, but importantly, also from an ethical point of view.

Research is increasingly focusing on therapy options to interrupt the self-perpetuating hyperinflammatory response by mechanical removal of inflammatory mediators and associated compounds. The rationale for adding the CytoSorb adsorber to a set of purification systems already in use was, on the one hand due to the continuous deterioration of the patient's clinical condition despite maximum standard therapy, but was also based on the knowledge that both CVVHD as well as therapeutic plasmapheresis are not able to eliminate significant amounts of inflammatory mediators from the blood compartment within a short period of time, and in the capacity needed [4]. On the contrary, CytoSorb acts through direct adsorption where the tightly structured network of polymer beads builds up a huge adsorption surface area that ensures removal of high amounts of various mediators including cytokines, chemokines, pathogenassociated molecular pattern molecules (PAMPs), damageassociated molecular pattern molecules (DAMPs) and toxins [9, 10].

Inflammatory cytokines play a crucial role in the pathogenesis of hyperinflammation and sepsis [11] and their plasma concentrations have been shown to be associated with outcome i.e. mortality is highest when both proinflammatory and anti-inflammatory cytokine levels are high [12, 13]. In our set of patients, eventual non-survivors showed pretreatment median levels for IL-6 and IL-10 of $1253.45 \mathrm{pg} / \mathrm{ml}$ and $826.9 \mathrm{pg} / \mathrm{ml}$, respectively, whereas survivors had significantly lower levels (IL-6 $18.9 \mathrm{pg} / \mathrm{ml}, \mathrm{IL}-100.5 \mathrm{pg} / \mathrm{ml}$ ). CytoSorb has been shown to effectively remove molecules in the middle molecular range (up to approx. $55 \mathrm{kDa}$ ), including cytokines, chemokines and various other substances in vitro and in animal models $[9,14]$, but has also proved effective in the clinical setting $[15,16]$. Generally higher levels of IL-10 and IL-6 in non-survivors is consistent with the finding that e.g. the persistence of IL- 6 levels in the blood rather than the peak levels per se is decisive for disease severity and outcome [17], which might be equal to IL-10 levels [13]. Therefore, IL-10 levels could possibly be considered a negative prognostic index and might be explained by the specific actions being the prototypical cytokine of the antiinflammatory system [18]. However, plasma concentrations of both IL-6 and IL-10 clearly decreased during the course of treatment, independent of eventual survival. In this context, high plasma concentrations were reduced more efficiently than low plasma levels, which is in line with the known concentration-dependent clearance properties of the CytoSorb adsorber. From a safety perspective, this lack of significant removal at physiologically necessary concentrations is important, as the body is dependent on a certain amount of cytokines to preserve an adequate immune response.

Hemodynamic stabilization with a concomitant reduction of catecholamine dosages but also milrinone is another prominent finding associated with the application of CytoSorb hemoadsorption. A recently published prospective single-center study by Friesecke and coworkers in twenty patients with septic shock refractory to standard treatment that were treated with CytoSorb showed a significant reduction in norepinephrine dosages and shock reversal in two thirds of the patients [19]. Likewise, patients undergoing cardiopulmonary bypass-assisted cardiac surgery (including those with endocarditis) who developed post-operative hyperinflammation, showed a marked benefit from hemoadsorption treatment in terms of hemodynamic stabilization and reduction in catecholamine dosages [2, 20].

Underlying mechanisms remain elusive, however it can be speculated that a lower cytokine load in the circulating blood results in less activation of vasoactive mediators (i.e. nitric oxide production via iNOS) resulting in stabilization of the vascular tone and a reduction in vasoplegia. Moreover, there is evidence, mainly from case reports, that hemoadsorption may have a positive impact on vascular barrier integrity and therefore indirectly on capillary leakage syndrome, which might also be part of the beneficial effects of CytoSorb therapy on hemodynamics [21-23].

We also found that timing was highly important in our set of patients. While all patients treated within 24 hours after diagnosis survived, none of those treated later than 48 hours did. This is in line with results from a case series in 26 critically ill adult patients conducted by Kogelmann et al [24]. They showed that patients in whom therapy was started early (within $<24 \mathrm{~h}$ ) exhibited an ICU mortality of $69.2 \%$, which was significantly lower than expected mortality $(92.3 \%)$. Likewise, patients with a therapy delay between $24-$ 48 hours had a better outcome than predicted. On the other hand, patients who were treated late ( $>48$ hours therapy delay) all died during their intensive care stay. It is well known, particularly in the field of sepsis, that the early onset of therapeutic measures is crucial to the outcome. In this context, early initiation of appropriate antimicrobial treatment as well as volume and catecholamine therapy has shown beneficial effects in large-scale clinical studies [25, 26]. The early onset of therapy might hinder the selfperpetuating loop of hyperinflammation to gain momentum. This early therapy start might be all the more important in children as they have a presumably higher potential for organ regeneration than older patients with numerous comorbidities. On the other hand, if therapy is initiated too late, vital organ functions might have already transitioned from a potentially reversible dysfunction all the way down to 
irreversibly manifested structural organ damage. However, more data is needed to determine the best therapeutic window including start and duration of therapy but also for the right indication. Additionally, the present case series is particularly important since it could be shown that the adult $300 \mathrm{ml}$ CytoSorb adsorber cartridge could be applied even in neonates without any technical issues and therefore has to be considered a safe treatment option also in young patients. Finally, by demonstrating the potential therapeutic effects of hemoadsorption, we highlight the need for the development and validation of a miniaturized system specifically dedicated to neonatal and pediatric patients.

\section{Limitations}

We are well aware of the fact that several limitations accompany this case series. First and most importantly, this is a case series only with no control cohort analyzing retrospective data. However, it represents the first ever description of the use of CytoSorb hemoadsorption in more than one pediatric patient. Moreover, data derived from this case series are in line with key findings from previous studies and therefore certainly contribute to increasing knowledge for the scientific community.

Second, we analyzed a very heterogeneous patient group with various indications, ages and treatments. For example, from the case series published by Kogelmann et al. there is evidence that medical patients seem to benefit more from hemoadsorption treatment than (post) surgical patients. Although CytoSorb has been applied in different etiologies and indications, predominantly in critically ill patients, its clinical effects (besides proven reduction of mediators from the blood) seem to be consistent and extend from hemodynamic stabilization accompanied by a reduction in catecholamines, to stabilization of vascular integrity and preservation of organ functions.

Third, the potential beneficial effect of hemoadsorption was tested in the context of a combined treatment with two other extracorporeal procedures for blood purification as well as in conjunction with other standard therapies, but also certain dedicated life support techniques (e.g. ECMO). Therefore, it is hard to determine the true contribution of CytoSorb in this complex clinical setting.

Fourth, data on clinically relevant outcome parameters such as days on mechanical support, CVVH etc. are hard to evaluate, and only serve for completeness of the dataset. While several non-survivors $(n=3)$ died early (between 4 and 7 days in ICU), a number of survivors had a relatively long stay in ICU and therefore had long times on extracorporeal support.

For all these reasons, this case series can be of a hypothesis-generating nature only. However, since this is a procedure not intended and/or routinely applied in pediatric patients, the results shown herein could help future trials answer specific questions particularly in pediatrics. Moreover, the present case series might be a trigger for the production and validation of a Cytosorb adsorber dedicated to the population of newborns and pediatric patients.

\section{Conclusions}

The use of Cytosorb seems to be effective in treatment of many sepsis related pathologies even in neonatal and pediatric patients. It is necessary to develop a dedicated sized Cytosorb to overtake the application problems related to age and weight of patients. Further prospective randomized controlled studies in the pediatric field are necessary to elucidate the full potential benefit of hemoadsorption in this set of patients.

\section{Conflicts of Interest and Source of Funding}

All the authors do not have any possible conflicts of interest.

\section{References}

[1] Rimmelé T, Kellum JA: Clinical review: blood purification for sepsis. Crit Care 2011;15:205.

[2] Träger K, Fritzler D, Fischer G, et al: Treatment of postcardiopulmonary bypass SIRS by hemoadsorption: a case series. Int J Artif Organs 2016;39:141-6.

[3] Datzmann T, Träger K: Extracorporeal membrane oxygenation and cytokine adsorption. J Thorac Dis 2018;10:S653-S660.

[4] Honore PM, Jacobs R, Joannes-Boyau O, et al: Newly designed CRRT membranes for sepsis and SIRS-- a pragmatic approach for bedside intensivists summarizing the more recent advances: a systematic structured review. ASAIO J 2013;59:99-106.

[5] Eyre M, Hacohen Y, Barton C, et al: Therapeutic plasma exchange in paediatric neurology: a critical review and proposed treatment algorithm. Dev Med Child Neurol 2018;60:765-779.

[6] Milella L: Neonatal and Pediatric General and Cardiac Anaesthesia and ICU: wath"s new in 2017/2018? -Bari Pediatric Hospital Experience-Italy." Journal of Pediatrics and Neonatal Care 2018;8:00309.

[7] Bonavia A, Groff A, Karamchandani K, et al: Clinical Utility of Extracorporeal Cytokine Hemoadsorption Therapy: A Literature Review. Blood Purif 2018;46:337-349.

[8] Milella L, Ficarella M: First Application of CVVHDF, Plasmapheresis and "Cytosorb a Bsorber" to Solve a Pediatric Haemophagocitic Histyocitosis Case. Res Pediatr Neonatol 2017;1: RPN.000510.

[9] Song M, Winchester J, Albright RL, et al: Cytokine removal with a novel adsorbent polymer. Blood Purif 2004;22:428-34

[10] Gruda MC, Ruggeberg KG, O'Sullivan P, et al: Broad adsorption of sepsis-related PAMP and DAMP molecules, mycotoxins, and cytokines from whole blood using CytoSorb ${ }^{\circledR}$ sorbent porous polymer beads. PLoS One 2018;13:e0191676. 
[11] Hotchkiss RS, Karl IE: The pathophysiology and treatment of sepsis. N Engl J Med 2003;348:138-50.

[12] Spittler A, Razenberger M, Kupper H, et al: Relationship between interleukin-6 plasma concentration in patients with sepsis, monocyte phenotype, monocyte phagocytic properties, and cytokine production. Clin Infect Dis 2000;31:1338-42.

[13] Kellum JA, Kong L, Fink MP, et al: Understanding the inflammatory cytokine response in pneumonia and sepsis: results of the Genetic and Inflammatory Markers of Sepsis (GenIMS) Study. Arch Intern Med 2007;167:1655-63.

[14] Kellum JA, Song M, Venkataraman R: Hemoadsorption removes tumor necrosis factor, interleukin-6, and interleukin10, reduces nuclear factor-kappaB DNA binding, and improves short-term survival in lethal endotoxemia. Crit Care Med 2004;32:801-5.

[15] Hetz H, Berger R, Recknagel P et al: Septic shock secondary to $\beta$-hemolytic streptococcus-induced necrotizing fasciitis treated with a novel cytokine adsorption therapy. Int $J$ Artif Organs 2014;37:422-6.

[16] Bruenger F, Kizner L, Weile J, et al: First successful combination of ECMO with cytokine removal therapy in cardiogenic septic shock: a case report. Int J Artif Organs 2015;38:113-6.

[17] Pinsky MR, Vincent JL, Deviere J, et al: Serum cytokine levels in human septic shock. Relation to multiple-system organ failure and mortality. Chest 1993;103:565-75.

[18] Saraiva M, O'Garra A: The regulation of IL-10 production by immune cells. Nat Rev Immunol 2010;10:170-81.
[19] Friesecke S, Stecher SS, Gross S, et al: Extracorporeal cytokine elimination as rescue therapy in refractory septic shock: a prospective single-center study. J Artif Organs 2017;20:252-259.

[20] Träger K, Skrabal C, Fischer G, et al: Hemoadsorption treatment of patients with acute infective endocarditis during surgery with cardiopulmonary bypass - a case series. Int $J$ Artif Organs 2017;40:240-249.

[21] David S, Thamm K, Schmidt BMW, et al: Effect of extracorporeal cytokine removal on vascular barrier function in a septic shock patient. J Intensive Care 2017;5:12.

[22] Hinz B, Jauch O, Noky T, et al: CytoSorb, a novel therapeutic approach for patients with septic shock: a case report. Int $J$ Artif Organs 2015;38:461-4.

[23] Träger K, Schütz C, Fischer G, et al: Cytokine Reduction in the Setting of an ARDS-Associated Inflammatory Response with Multiple Organ Failure. Case Rep Crit Care 2016;9852073.

[24] Kogelmann K, Jarczak D, Scheller M, et al: Hemoadsorption by CytoSorb in septic patients: a case series. Crit Care 2017;21:74

[25] Kumar A, Roberts D, Wood KE, et al: Duration of hypotension before initiation of effective antimicrobial therapy is the critical determinant of survival in human septic shock. Crit Care Med 2006;34:1589-96.

[26] Rivers E, Nguyen B, Havstad S, et al: Early Goal-Directed Therapy Collaborative Group. Early goal-directed therapy in the treatment of severe sepsis and septic shock. $N$ Engl J Med 2001;345:1368-77. 